\title{
FÁBRICA ACADÉMICA DE SOFTWARE
}

\author{
Zalatiel Carranza Avalos \\ Universidad de Lima
}

\section{Resumen}

En los últimos años, cada vez con mayor frecuencia, se ha escuchado hablar en el medio profesional de la informática, de "fábricas de software". Al mismo tiempo, el hecho de que algunas universidades que poseen facultades de sistemas tengan áreas dedicadas a la ingeniería de software, ha incentivado la realización de estudios para aclarar el significado de dicha expresión, así como la discusión sobre la posibilidad de ampliar su significado con el fin de darle un uso académico y establecer los requerimientos para implementar una fábrica académica de software en la Facultad de Ingeniería de Sistemas de la Universidad de Lima.

Una vez culminada la investigación bibliográfica, se realizaron algunas entrevistas a gerentes de fábricas de software de nuestro medio y se presentó un informe preliminar, que fue el punto de partida de la discusión sostenida en un focus group, en el que participaron los gerentes de las fábricas de software más representativas de Lima.

Los resultados de esta investigación han confirmado que la expresión fábrica de software es un analogía que trata de expresar la conveniencia de copiar las mejores prácticas usadas en diferentes industrias que fabrican bienes, para utilizarlas en el proceso de desarrollo de software.

Palabras clave:

fábrica de software, desarrollo de software, normas y estándares de calidad del software, procesos de construcción del software, proyectos de software 


\section{Introducción}

Los profesionales que laboran en el área de software y aquellos que algo conocen sobre el tema, saben que el software no se fabrica, como ocurre con los computadores, los autos o las camisas, sino que el software se desarrolla, es decir que, a diferencia de los computadores, que requieren el diseño previo de un prototipo para luego pasar a implementar una línea de producción en masa, en el caso del software hay que definir los requerimientos, hacer el análisis, el diseño, la construcción, las pruebas y la instalación o despliegue, debido a que no hay una producción en masa propiamente dicha.

En el caso de los computadores, cuando hay una falla en el producto, hay que realizar ajustes en el diseño y en la línea de producción. En el caso del software, de existir una falla habrá que efectuar ajustes en las etapas pertinentes del desarrollo del software antes mencionadas, en un proceso que resulta normalmente menos complicado y costoso que en el primer proceso.

Las primeras investigaciones dieron como resultado que en 1998 se ponga en marcha una fábrica académica de software, como parte de un taller de ingeniería de software en la Pontificia Universidad Católica de Chile.

En el 2007, en la Universidad de Lima se realizaron estudios sobre el concepto de fábrica de software, así como su adaptación para darle un uso académico. Este mismo año se intercambiaron ideas con los gerentes de diversas fábricas de Lima y los primeros meses del 2008 se realizaron focus group a los que asistieron los representantes de las fábricas de software más importantes del país.

El principal resultado de esta investigación permitió establecer los requerimientos de una fábrica académica de software para la Facultad de Ingeniería de Sistemas de la Universidad de Lima.

\section{Conceptos sobre fábrica de software}

Como se mencionó líneas arriba, el software no se fabrica, y la expresión "fábrica de software" es una analogía que trata de expresar la necesidad de copiar las buenas prácticas de las fábricas que producen bienes para tratar de desarrollar software con un proceso cada vez menos artesanal.

Este concepto es antiguo y nuevo a la vez. Es antiguo porque el vocablo expresión "fábrica de software" apareció en 1968; sin embargo, al mismo tiempo es nuevo, pues su significado ha ido cambiando con el 
progreso de la tecnología y la aparición de nuevas áreas de conocimiento, por ejemplo: "control de calidad", "marcos de las mejores prácticas para la gobernabilidad de la tecnología de información", "gestión de proyectos", entre otras.

No hay una definición compartida por todos los entendidos sobre lo que es fábrica de software; sin embargo, parece que todos están de acuerdo respecto a que este concepto incluye el establecimiento de procesos definidos con el objeto de acelerar el desarrollo reusando componentes para mejorar la calidad del software producido. La meta en todos los casos es la maximización de la efectividad de recursos, la estimación precisa y la minimización del tiempo y los costos, así como la mejora continua de la calidad de sus procesos y de sus productos.

Posiblemente, la gran diferencia de opinión entre los entendidos en el tema se deba a que algunos consideran que una fábrica de software debe ser especializada en el desarrollo de un tipo particular de software y, por lo tanto, su plataforma de desarrollo y sus procedimientos cambian muy poco. Otra corriente de pensamiento, compartida por no pocos profesionales, no establece tal limitación, permitiendo contar con fábricas de software con varias líneas de producción para desarrollar cualquier tipo de producto con una gran flexibilidad de plataforma y variantes de sus procedimientos.

En cualquier caso, cada línea de producción de una fábrica de software contiene tres ideas claves: un esquema de fabricación, que es equivalente a un proceso o a una receta para desarrollar software; los elementos de su construcción, que son bloques de construcción prehechos para aprovechar las ventajas de reúso, y un ambiente de desarrollo, que son las plataformas tanto de software como de hardware utilizadas para desarrollar dicho software.

\section{Historia de la fábrica de software}

La expresión "fábrica de software" aparece por primera vez en una propuesta presentada por Robert William Bemer en el congreso de la International Federation of Information Processing (IFIP) en 1968. Bemer (1920-2004) fue un informático conocido por sus trabajos en IBM durante los últimos años de la década de 1950 y principios de la de 1960; antes había laborado para Rand Corporation y desde 1974 trabajó en Honeywell. Coincidiendo con el boom de la informática de consumo en los años sesenta, Bemer inventó, como parte del código ASCII, en cuya comisión de definición participó, la tecla Esc, más conocida como Escape, que sirve 
para detener o cancelar la mayoría de los programas o aplicaciones informáticas. Por esta razón se le conoce a veces como el padre del ASCII. Bemer, no obstante, es más conocido por otras contribuciones al mundo de la informática. En 1959 participó en el desarrollo del lenguaje Cobol. Este ingeniero también fue el primero en alertar sobre las consecuencias del efecto del año 2000, pero nadie lo tomó en serio.

En 1969, Hitachi instaló la primera fábrica de software; Rand Corporation instaló la segunda en 1976, luego lo hicieron NEC en 1976, Toshiba en 1977, Fujitsu en 1979, NTT en 1985 y Mitsubishi en 1987.

En la década de 1990 aparecen fábricas basadas en entornos de desarrollo integrados, en componentes, en la madurez de procesos, en la reutilización y renovación de software, y enfocadas a otras técnicas de gestión de la calidad. A partir del 2000, utilizando las últimas áreas de conocimientos, aparecen las fábricas basadas en componentes, modelos y líneas de productos.

En nuestro país hay algunos esfuerzos para mejorar la calidad de los procesos para desarrollar software en las más importantes empresas dedicadas a esta labor. Las más notables son IBM, que cuenta con una certificación CMMI a nivel 5 corporativo, el Banco de Crédito del Perú (CMMI nivel 3), GMD (CMMI nivel 3), CosapiSoft (CMMI nivel 3). Mientras importantes empresas como Novatronic, Sinopsis, y Avatar, entre otras, están realizando la mejora de sus procesos para desarrollar software.

\section{Características de los procesos de la fábrica de software}

Los procesos de una fábrica de software tienen las siguientes características: configuran, adaptan y ensamblan rápidamente componentes; usan patrones de industrialización, estándares, actividades repetibles y automatizables; miden el desempeño y la productividad de las personas que participan en el desarrollo del software; utilizan la planificación para controlar el desarrollo acelerado del software; y sus actividades son establecidas con criterios de rentabilidad, tratando de obtener presupuestos y plazos predecibles, así como una calidad aceptada en el ámbito mundial.

Construir un producto software utilizando una fábrica de software implica las siguientes actividades: establecer los requerimientos del producto en coordinación con el usuario, realizar un análisis para indicar qué hará el producto, y luego especificarlo formalmente para pasar a diseñarlo y a establecer los componentes que se pueden volver a usar en su construcción. Terminado el diseño se puede pasar a la implementación, despliegue y prueba del producto. 


\section{Conclusión sobre la fábrica de software}

Como resultado del primer objetivo de la investigación: definir y entender el concepto de fábrica de software, se puede concluir que la expresión "fábrica de software" es una analogía que trata de enfatizar las ventajas de un nuevo enfoque para desarrollar software.

Las fábricas de software seleccionan componentes, los ajustan, los adaptan, los amplían y ensamblan dichos componentes; intentan aprender de otras industrias que encaran problemas similares y aplican patrones específicos de automatización; vuelven más rápida, barata y fácil la construcción de aplicaciones y desarrollan productos de software con rapidez y calidad a través de procesos conocidos, repetibles y gerenciables, $y$, sobre todo, mejorables continuamente.

\section{Fábrica académica de software}

\subsection{Definición de fábrica académica de software}

Una fábrica académica de software es similar a una fábrica de software empresarial, pero su objetivo es servir como medio de aprendizaje. Emplea estándares internacionales de calidad, trata la parte productiva de software de una organización y soporta varias líneas de producto. Pero no considera las áreas convencionales de las organizaciones, como administración, finanzas, comercialización y otras similares.

El fin de una fábrica académica de software es mostrar cómo se elabora software de calidad y cómo es producido en una organización dedicada a esta actividad. Permite la construcción del software como un proceso industrial, definible, repetible y medible, en el que la construcción de un producto no depende del conocimiento de sus constructores.

\subsection{Ventajas de los usuarios de una fábrica académica de software}

Los usuarios de una fábrica académica de software de la facultad de una universidad cuyo plan de estudios incluye temas relacionados con el desarrollo de software serán los alumnos, los egresados y los profesores de dicho centro de estudios.

Los alumnos y egresados podrán practicar la implementación de normas y modelos de calidad de software de carácter internacional, aplicar e integrar conocimientos teóricos para resolver problemas reales y con- 
tar con una biblioteca de componentes ejecutables, incluyendo patrones de diseño de software en lenguajes de programación como . Net y Java.

Los profesores tendrán plataformas de hardware y software para crear los casos prácticos que integren conocimientos de diversas asignaturas. Así mismo, se podrán usar estas instalaciones para brindar servicios a empresas interesadas en los productos que dicha fábrica pueda elaborar.

\subsection{Pasos para crear una fábrica académica de software}

Para crear una fábrica académica de software se deben ejecutar los siguientes pasos:

- Definir y entender qué es una fábrica académica de software, pues es importante que los miembros de la institución conozcan las facilidades de las que dispondrán para aprovecharlas de la mejor manera.

- Establecer la organización y perfil de roles requeridos. Como cualquier organización, se debe definir el organigrama de las áreas funcionales con que contará la fábrica, y, así mismo, se deben definir las características de los roles requeridos en los distintos cargos necesarios para su funcionamiento.

- Seleccionar marcos de arquitectura y modelos de calidad. Dependiendo de los distintos tipos de productos por desarrollar, es posible que se utilicen marcos arquitectónicos adecuados para cada caso; por ello, habrá que determinar qué marcos arquitectónicos se han de usar, así como qué modelos de calidad serán requeridos en cada caso.

- Determinar la ubicación de la fábrica de software. En este lugar se instalarán los equipos y será el área de trabajo de los que laboran en dicha fábrica.

- Establecer una línea de producto inicial. Lo más probable es que la fábrica empiece produciendo un particular tipo de software; por ello, debe elegirse dicha línea para preparar lo que sea necesario con el fin de iniciar el funcionamiento de la fábrica.

- Determinar los procesos que se utilizarán. Siguiendo algún modelo estándar, habrá que especificar los procesos y procedimientos para el funcionamiento de la fábrica.

- Especificar las plataformas de hardware y software que se utilizarán. Las líneas de producción de software por desarrollarse necesitan plataformas básicas de hardware y software, que inicialmente no requie- 
ren de grandes inversiones. Dichas plataformas se irán ampliando en la medida en que se justifique su crecimiento.

- Obtener la aprobación de las autoridades. No hay duda de que un proyecto tan importante, y sobre todo por los recursos requeridos, aunque no son muy costosos, debe contar con la aprobación de las autoridades correspondientes.

- Contar con personal del perfil requerido. Para iniciar el funcionamiento de la fábrica académica de software será necesario contar con personal que desempeñe los roles previstos, para lo que habrá que seleccionar profesores y alumnos practicantes que reúnan las certificaciones correspondientes.

- Instalar plataformas de hardware y software. El funcionamiento de una fábrica académica de software está supeditado a la existencia de las plataformas de hardware y software instaladas y funcionando para que el personal encargado pueda iniciar el desarrollo de los productos de software solicitados por los usuarios.

- Ofrecer servicios a los interesados. La fábrica académica de software solo se justifica por la existencia de sus usuarios, de modo que tan pronto se cuente con los recursos antes mencionados debe iniciar sus operaciones, fabricando los productos de software requeridos por los interesados.

\section{Requerimientos de una fábrica académica de software para la Facultad de Ingeniería de Sistemas de la Universidad de Lima}

\subsection{Roles requeridos por una fábrica académica de software}

Los roles tendrán una organización que puede variar dependiendo de la orientación que tenga la fábrica de software correspondiente. No hay duda de que se requiere un director de la fábrica de software, del cual dependerán: un jefe de Desarrollo, un jefe de Implementación, un jefe de Despliegue y un jefe de Normas.

Es deseable que los roles de jefe sean desempeñados por profesores de los cursos relacionados con los temas correspondientes. Algunos roles de menor nivel deben ser desempeñados por alumnos practicantes. El personal deberá contar con certificaciones en herramientas de desarrollo de software en marcos, modelos y estándares de calidad internacionales. 


\subsection{Marcos, modelos, estándares de calidad y herramientas requeridos por una fábrica académica de software}

Los procesos por implantar deben basarse en marcos, modelos y estándares de calidad internacionales como el marco ITIL, la norma ISO/IEC 12207, el modelo CMMI, la norma Spice (ISO/IEC 15504), el marco PMBOK, el marco Cobit, la norma 17799, la norma ISO9000, la metodología Six Sigma y otros.

Las herramientas para apoyar los procesos de desarrollo de software pueden incluir Requisite pro de IBM Racional de IBM, Project Server de Microsoft, Clarity de Computer Associates, ProactiveOffice de la empresa del mismo nombre, PEIS de Cofre Consultores, WS Charp-pro de $\mathrm{E}+\mathrm{PMC}$, entre otros.

\subsection{Ubicación física, plataformas de hardware y software de la fábrica académica de software}

Como se ha mencionado anteriormente, una fábrica de software requiere un lugar específico para su funcionamiento. En el caso de la Facultad de Ingeniería de Sistemas de la Universidad de Lima, la fábrica podría ser el Laboratorio de Tecnología de Información, ubicado en el primer sótano del pabellón $W$.

La plataforma de hardware podrá contar con equipos de diferentes tipos, de acuerdo con las necesidades; por ejemplo, equipos del tipo 1, que incluyen servidores Intel Dual Core 4GB RAM mínimo recomendado, disco duro 120GB SCSI; interfaces de red, lectora de DVD-RW utilizada tanto para desarrollo como para producción. También serán necesarios equipos tipo 2, que incluyen servidores Intel Dual Core 2GB RAM, disco duro 120GB SCSI, interfaces de red y lectora DVD-RW para desarrollo. Asimismo, pueden usarse equipos tipo 3, que comprenden estaciones de trabajo Dual Core, 2GB RAM, mínimo recomendado, disco duro 100GB, interfaces de red y lectora DVD-R para desarrollo. Finalmente, se pueden incluir equipos tipo 4, que pueden considerar estaciones de trabajo Dual Core, 1 GB RAM, disco duro $80 \mathrm{~GB}$, interfaces de red y lectora DVD-R para ofimática.

La plataforma de software contará con software de máquinas virtuales con ambientes que brindan: servicios de sistemas operativos, servicios de gestión de datos, servicios de intercambio de datos, servicios de ingeniería de software, servicios de desarrollo de software, servicios de comunicaciones y servicios de seguridad. 


\section{Focus group sobre fábrica académica de software}

Con el objetivo de discutir los conceptos sobre fábrica académica de software, validar los resultados obtenidos de este proyecto y tomar nota de las sugerencias de los expertos en el tema, se realizó el 13 de marzo del 2008, en la Universidad de Lima, un conversatorio sobre el tema, con la participación de:

- David Muñoz, director gerente general de Avantica.

- Ricardo Bustamante, gerente de Sistemas del Banco de Crédito del Perú.

- Tobías Aliaga, gerente de Soluciones Empresariales de Cosapi Data.

- Aldo Galli, gerente de División de Procesos de Negocios, GMD.

- Ricardo Haro, de Technical Solution Manager Global Business Services - Application Services, IBM del Perú.

- Rolando Liendo, gerente general de Lolimsa.

- Ronald Armas, especialista en arquitectura de Soluciones de Microsoft Perú S.R.L.

- Guillermo Pacheco, director gerente de Novatronic.

Durante el conversatorio sostenido con los gerentes se expuso el proyecto y se procedió al focus group, cuyos enunciados, respuestas y comentarios se transcriben a continuación:

1. Respecto a la conclusión presentada sobre una fábrica de software usted está:

$\begin{array}{ll}\text { Totalmente de acuerdo } & 25,0 \\ \text { Bastante de acuerdo } & 62,5 \\ \text { Medianamente de acuerdo } & 12,5 \\ \text { Poco de acuerdo } & 00,0 \\ \text { Totalmente en desacuerdo } & 00,0\end{array}$

Es importante observar que si bien los que están totalmente de acuerdo solo alcanza el 25\%; sin embargo, los gerentes invitados estuvieron de acuerdo con dicha conclusión en un $62,5 \%$ y solo el $12,5 \%$ estuvo medianamente de acuerdo con el resultado, lo cual nos confirma que la 
definición de lo que es una fábrica de software depende mucho de la manera como cada institución ha adaptado el concepto según sus propias necesidades. Es decir que algunos gerentes usan la fábrica de software para producir software a medida y otros para producir un tipo de software particular, lo que hace que sus requerimientos sobre lo que debe ser una fábrica de software sean distintos.

Algunos participantes enfatizaron la necesidad de incluir la gestión del conocimiento y establecer que la automatización de la línea de producción era un aspecto básico de una fábrica de software. Asimismo, se recomendó que el alcance de la fábrica de software tome en cuenta todas las etapas de la vida del software, incluyendo el mantenimiento.

2. La implementación de una fábrica académica de software en la Facultad de Ingeniería de Sistemas de la Universidad de Lima es un tema:

$\begin{array}{lc} & \text { Porcentaje } \\ \text { Muy importante } & 37,5 \\ \text { Importante } & 50,0 \\ \text { Medianamente importante } & 12,5 \\ \text { Poco importante } & 00,0 \\ \text { Sin importancia. } & 00,0 .\end{array}$

Sobre estas cifras, los asistentes recomendaron que se implemente la fábrica académica de software y que se le utilice para realizar aprendizaje usando casos que puedan ser desarrollados en varios ciclos, enfatizando el uso de procesos para garantizar que los egresados de la Facultad de Ingeniería de Sistemas de la Universidad de Lima tengan una experiencia real del trabajo que pueden realizar, si optan por esta área laboral, al terminar sus estudios. Esta puede ser una manera de vincular la empresa y la universidad a través de proyectos de desarrollo de software conjuntos.

Sobre la denominación de la fábrica algunos asistentes recomendaron el nombre "Centro de desarrollo" y otros "Centro de Soluciones de Tecnología de Información". 
3. Respecto a los pasos para crear una fábrica académica de software: ¿Tiene alguna sugerencia sobre algo que se deba agregar? Las sugerencias fueron:

a. La estructura organizacional es muy importante.

b. Los procesos repetibles deben asociarse a los diferentes enfoques de desarrollo.

c. Es muy importante una variedad de plataformas.

d. Tomar en cuenta el enfoque de proyectos.

4. ¿Tiene alguna sugerencia sobre el orden de los pasos?

Sobre esta consulta no hubo ninguna sugerencia, más bien se confirmó que el orden propuesto era el adecuado.

5. ¿Cuáles son los marcos, modelos y estándares de calidad que usa su empresa actualmente?

El modelo $\mathrm{CMMI}$

EI PMBOK

Otros marcos, modelos, estándares

El marco ITIL

La norma 17799

La norma ISO/IEC 12207

El marco Cobit

La norma ISO-9000

La metodología Six Sigma

La norma Spice (ISO/IEC 15504)

\section{Porcentaje}

100,0

87,5

75,0

62,5

62,5

50,0

50,0

37,5

25,0

12,5

Esta encuesta confirma que los gerentes de las fábricas de software invitados consideran que CMMI es el modelo más adecuado para la gestión de la calidad y que PMBOK es la referencia más adecuada para el manejo de proyectos de software. Por otro lado, esta encuesta también nos indica que la norma ISO-9000, la metodología Six Sigma y la norma Spice (ISO/IEC 15504) no son muy usadas en nuestro medio para la gestión de fábricas de software. 
6. ¿Cuáles son los marcos, modelos y estándares de calidad que usará su empresa en los próximos dos años?

$\begin{array}{lc} & \text { Porcentaje } \\ \text { El modelo CMMI } & 100,0 \\ \text { El PMBOK } & 87,5 \\ \text { Otros marcos, modelos, estándares } & 75,0 \\ \text { El marco ITIL } & 62,5 \\ \text { La norma 17799 } & 62,5 \\ \text { La norma ISO/IEC 12207 } & 50,0 \\ \text { El marco Cobit } & 50,0 \\ \text { La norma ISO9000 } & 37,5 \\ \text { La metodología Six Sigma } & 25,0 \\ \text { La norma Spice (ISO/IEC 15504) } & 12,5\end{array}$

Esta encuesta confirma que los gerentes invitados consideran que CMMI es el modelo más idóneo para la gestión de la calidad y que PMBOK es la referencia más adecuada para el manejo de proyectos de software para los próximos dos años. Por otro lado, la encuesta también indica que la norma ISO-9000, la metodología Six Sigma y la norma SPICE (ISO/IEC 15504) no serán muy usadas en nuestro medio para la gestión de fábricas de software durante los dos siguientes años. Es decir, los gerentes de fábricas de software asistentes al focus group no avizoran cambio alguno de marcos, modelos y estándares de calidad en los dos próximos años.

7. Respecto a las herramientas por utilizar, ¿tiene alguna sugerencia sobre algo que se deba agregar?

Las sugerencias enfatizaron la posibilidad de usar herramientas de software libre, entre ellas Ruby on Rails. También se sugirieron herramientas como:

- Domain Specific Languages Toolkit, para ser usada sobre plataforma Microsoft.

- Mercury Testing Software.

- Harvest/All Fusion. 
8. ¿Tiene alguna sugerencia sobre algo que se debe retirar? Al respecto, no hubo sugerencia concreta alguna.

9. Con relación a las fuentes para la implementación de patrones, la sugerencia fue utilizar Patterns and Practices de Microsoft.

Los comentarios finales de los participantes fueron los siguientes:

- No perder de vista que un ingeniero de sistemas debe ser capaz de comunicarse en inglés.

- Considerar la posibilidad de usar la fábrica académica de software para realizar investigaciones para empresas o con fondos de organismos internacionales que apoyan la investigación y el desarrollo.

- La Universidad de Lima debe efectuar reuniones, como la realizada en esta oportunidad, con el fin de intercambiar ideas con la empresa para concretar acuerdos de mutuo beneficio.

- Es muy importante la capacitación de los alumnos en el tema de procesos.

- La fábrica de software debe propiciar el uso en vivo de la tecnología.

- No debe perderse de vista el concepto de negocio al hacer uso del concepto fábrica de software.

- El concepto de calidad tiene que estar presente de una manera muy relevante en una fábrica de software.

\section{Conclusiones de la investigación}

- La expresión "Fábrica de software" es una analogía que trata de enfatizar las ventajas de un nuevo enfoque para desarrollar software.

- Las fábricas de software en general:

- Seleccionan componentes, los ajustan, los adaptan, los amplían y los ensamblan.

- Intentan aprender de otras industrias que encaran problemas similares y aplican patrones específicos de automatización.

- Vuelven más rápida, barata y fácil la construcción de aplicaciones. 
- Producen con rapidez y calidad a través de procesos conocidos, repetibles y gerenciables, y, principalmente, factibles de mejoras continuas.

- Es posible adaptar el concepto de fábrica de software empresarial para aplicarlo al ambiente académico, teniendo en cuenta al hacerlo que sus fines ya no son económicos, sino servir como medio de enseñanza de los aspectos productivos de software de una organización y soportar varias líneas de producto, permitiendo la construcción del software como un proceso industrial, definible, repetible y medible, en el que el proceso de construcción de un producto no depende del conocimiento de sus constructores.

- La implementación de una fábrica académica de software en la Facultad de Ingeniería de Sistemas de la Universidad de Lima, por su aporte al mejor aprendizaje del desarrollo de software de los alumnos, constituye una ventaja competitiva respecto a otras facultades de otras universidades que ofrecen carreras similares. De esta manera, se implementará una fábrica de software, con características similares a las que existen en el ambiente empresarial, para que los alumnos de dicha facultad apliquen e integren los conocimientos teóricos recibidos en el conjunto de asignaturas de su Plan de Estudios.

- Establecer una fábrica académica de software puede permitir a los alumnos y profesores de la Facultad de Ingeniería de Sistemas realizar investigaciones para empresas $u$ otras con fondos proporcionados por organismos internacionales que apoyan la investigación y desarrollo.

- Los participantes en el focus group organizado con el fin de conocer la opinión de los gerentes de las fábricas de software más representativas de nuestro medio manifestaron lo siguiente:

- Estar de acuerdo con la conclusión sobre fábrica de software expresado en esta investigación.

- Que la definición de lo que es una fábrica de software depende mucho de la manera en que cada institución ha adaptado del concepto de acuerdo a sus propias necesidades.

- Que el alcance de la fábrica de software debe tomar en cuenta todas las etapas de la vida del software, incluyendo el mantenimiento.

- Que el tema de una fábrica académica de software es muy importante para una facultad de sistemas de una universidad, en opinión del $37,5 \%$ de los participantes al focus, mientras que el $50 \%$ consideró que es un tema importante. 
- Los gerentes de las fábricas de software de Lima consideran que CMMI es el modelo más adecuado para gestión de la calidad y que PMBOK es la referencia más adecuada para el manejo de proyectos de software.

\section{Recomendaciones de la investigación}

- Debido a la ventaja competitiva que representa frente a otras facultades de ingeniería de sistemas y de ramas afines de otras universidades, es muy recomendable la implementación de una fábrica académica de software en la Facultad de Ingeniería Sistemas de la Universidad de Lima. Esta implementación permitirá la participación de profesores que enseñan las distintas asignaturas del Plan de Estudios, en especial los del área de Ingeniería de Software, aportando cada uno su visión de cómo organizar los proyectos, en concordancia con el contenido de las asignaturas que dictan, para desarrollar proyectos multidisciplinarios, que son necesarios para la formación profesional de los alumnos.

- Otra razón que hace recomendable la implementación de una fábrica académica de software en la Facultad de Ingeniería de Sistemas de la Universidad de Lima es que su puesta en marcha no implica una inversión de recursos económicos importantes, sino más bien organizar los recursos humanos existentes, conformados por profesores y alumnos.

- Asimismo, es recomendable su ejecución con el fin de apoyar el desarrollo de las pequeñas empresas, que encontrarían en la Facultad de Ingeniería de Sistemas un laboratorio que los ayude a organizar sus propias fábricas de software.

- Se deben realizar focus groups con la participación de profesionales destacados de los grupos de interés correspondientes, con el fin de validar proyectos de investigación y el contenido de las nuevas asignaturas del Plan de Estudios de la Facultad de Ingeniería de Sistemas, sobre todo cuando los temas por tratar no están completamente maduros. 


\section{Bibliografía}

Braude, Eric J. Ingeniería de software: Una perspectiva orientada a objetos. México: Alfaomega, 2003.

Piattini Velthuis, Mario Gerardo. Calidad en el desarrollo y mantenimiento del software. México: Alfaomega, 2003.

Pressman, Roger S. Ingeniería del software: Un enfoque práctico. 6. ${ }^{a}$ edición. México: McGraw-Hill, 2005.

Schach, Stephen R. Ingeniería de software clásica y orientada a objetos. 6. ${ }^{a}$ edición. México: McGraw-Hill, 2006.

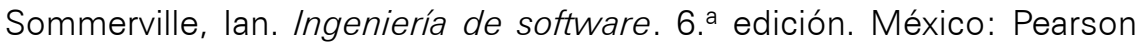
Educación/Addison Wesley, 2002.

\section{Páginas web}

Accesogroup. 10 pasos para crear una fábrica de software en España [en línea].<http://www.Accesomedia.Com/Display_Release.Html?ld= 29369 > . [Consulta: 20 de julio del 2007.]

Garzás Javier de Kybele Consulting. Primeras fábricas software, concepto e historia. [en línea]. <http://Kybeleconsulting.Blogspot.Com/ 2007/05/Primeras-Fábricas-Software-Concepto-E.Html>. [Consulta: 20 de junio del 2007.]

Microsoft. Un caso para la fábrica de software [en línea]. <http:// www.Microsoft.Com/Spanish/Msdn/Articulos/Archivo/041206/Noic es/Thecaseforsf.Mspx>. [Consulta: 8 de agosto del 2007.]

Fábrica de software. Creando software de calidad (2004-2007) [en línea]. $<$ http://www.Fabricadesoftware.CI/Viewforum.Php?F=20\&Sid= 7c8533954c4905507c0c42e9caf0dde1 >. [Consulta: 16 de abril del 2007.]

Universidad Autónoma de México. La industria del software, una oportunidad para México [en línea]. <http://www.Enterate.Unam.Mx/ Articulos/2002/Enero/Software.Htm>. [Consulta: 17 de abril del 2007]. 